\title{
Equilibrium Selection and Consistency
}

\author{
Henk Norde, Jos Potters, Hans Reijnierse, and Dries Vermeulen \\ Department of Mathematics, University of Nijmegen, \\ The Netherlands
}

Received October 24, 1994

\begin{abstract}
In this paper we show that, for two important classes of strategic games, namely the class of mixed extensions of finite games and the class of games with compact and convex strategy spaces and continuous-concave payoff functions, equilibrium selection is incompatible with One Person Rationality, Consistency and (restricted) Non-Emptiness. ๑1996 Academic Press, Inc.
\end{abstract}

\section{INTRODUCTION}

For two important classes of strategic games, the existence of Nash equilibria has been shown. The first class is the class of mixed extensions of finite games (Nash, 1951). The second class is the family of strategic games with compact convex strategy spaces and continuous payoff functions with the property that the payoff function of each player is concave in his strategy (Glicksberg, 1952). In fact, in many cases the set of Nash equilibria is too large and contains-from a strategic point of view-unreasonable equilibria (e.g. equilibria using weakly dominated strategies). This observation has been the starting point of the theory of equilibrium selection (cf. Harsanyi and Selten, 1982) as well as the theory of refinements (cf. Van Damme, 1987) and stable sets (cf. Kohlberg and Mertens, 1986). These theories have in common that they try to find for each game in a certain class of strategic games one equilibrium (equilibrium selection) or a nonempty subset of Nash equilibria (refinements or stable sets) as a collection of more advisable equilibria. For the two classes mentioned before we will prove that these attempts are bound to lead to inconsistent solutions.

So we will investigate solution rules $\varphi$ defined on the class $\Gamma_{\mathrm{f}}$ of all mixed extensions of finite games or defined on the class $\Gamma_{c}$ of games wherein the 
strategy spaces are convex and compact subsets of finite-dimensional vector spaces and the payoff functions are continuous-concave functions. We assume that the solution rule $\varphi$ assigns to each game $G \in \Gamma_{\mathrm{f}}$ (or $\Gamma_{\mathrm{c}}$ ) a nonempty set of strategy profiles (NEM) and to each one-person game a subset of the set of strategies where the payoff function takes its maximum (UM). We shall prove that $\varphi$ assigns to each game $G \in \Gamma_{\mathrm{f}}$ (or $\Gamma_{\mathrm{c}}$ ) the Nash equilibrium set $\operatorname{NE}(G)$, if $\varphi$ satisfies, moreover, the consistency property introduced by Peleg and Tijs (1992).

First we recall the definitions of reduced game and a consistent solution concept.

Let $G:=\left\{N^{G},\left\{A_{i}, U_{i}\right\}_{i \in N^{G}}\right\}$ be a strategic game in a class $\Gamma$ of strategic games, let $x$ be a strategy profile in $G$ and let $S$ be a proper subcoalition of the player set $N^{G}$ of $G$ (i.e., $S \neq N^{G}, \varnothing$ ).

The reduced game $G^{S, x}$ of the game $G$ with respect to $S$ and $x$ is a strategic game with player set $S$. Each player $i \in S$ has the same strategy space $A_{i}$ as in $G$ and the payoff functions $\left\{U_{i}^{\prime}\right\}_{i \in S}$ are defined by $U_{i}^{\prime}\left(y_{S}\right)$ : = $U_{i}\left(y_{S}, x_{M S}\right)$ for every strategy profile $y_{S}$. In the reduced game $G^{S, x}$ the players outside $S$ stick upon their strategies in $x$ and only the players in $S$ are free to reconsider their choice.

A solution rule $\varphi$ defined on $\Gamma$ is called consistent (CONS) if for every game $G \in \Gamma$, every proper coalition $S \subset N^{G}$ and every element $x \in \varphi(G)$,

$$
\text { (i) } G^{S, x} \in \Gamma, \quad \text { (ii) } x_{S} \in \varphi\left(G^{S, x}\right) \text {. }
$$

So, under a consistent solution rule the players of a reduced game do not need to reconsider their strategy choice: they can play the same strategy as in the original game.

The main theorem of the paper states that there is only one solution rule $\varphi$ defined on $\Gamma_{\mathrm{f}}\left(\right.$ or $\Gamma_{\mathrm{c}}$ ), satisfying non-emptiness (NEM), utility maximizing for 1-person games (UM), and consistency (CONS): the Nash equilibrium solution NE.

THEOREM 1. If $\Gamma=\Gamma_{\mathrm{f}}$ or $\Gamma_{\mathrm{c}}$ and $\varphi$ is a solution rule defined on $\Gamma$ with the properties (NEM) (i.e., $\varphi(G) \neq \varnothing$ for all $G \in \Gamma)$, (UM) (i.e., $\varphi(G) \subset$ $\arg \max U$ if $G$ is a 1-person game in $\Gamma$ and $U$ is the payoff function) and (CONS), then $\varphi(G)=\operatorname{NE}(G)$ for all games $G \in \Gamma$.

Note that the theorem gives not only a characterization of the Nash equilibrium solution on $\Gamma_{\mathrm{f}}$ and $\Gamma_{\mathrm{c}}$; it also frustrates beforehand every attempt to find a solution rule for $\Gamma_{\mathrm{f}}$ or $\Gamma_{\mathrm{c}}$ (unequal to NE) that extends the idea of utility maximization of 1-person games, assigns to each game a nonempty subset of strategy profiles, and satisfies consistency. All (proper) refinements of the Nash equilibrium solution are therefore violating consistency or nonemptiness. As a consequence of the theorem, perfectness, 
properness, and persistency must give inconsistent solution rules, as there are existence theorems for these types of Nash equilibria (cf. Selten, 1975; Myerson, 1978; and Kalai and Samet, 1984). Quasi-strictness (cf. Harsanyi, 1973), strongness (cf. Aumann, 1959) and coalitional proofness (Bernheim et al., 1987) are consistent solution rules satisfying (UM) (this is easy to check for quasi-strictness; see Peleg and Tijs (1992) for the last two solution rules) and must therefore violate nonemptiness on $\Gamma_{\mathrm{f}}$. Of course these results are not new but what is new is that they all follow immediately from one theorem.

In the following proposition we prove that every solution rule defined on any class of strategic games that satisfies (UM) and (CONS) is a subsolution of the Nash equilibrium solution.

Proposition 2. If $\varphi$ is a solution rule defined on a class $\Gamma$ of strategic games and $\varphi$ satisfies (UM) and (CONS), then $\varphi(G) \subset \mathrm{NE}(G)$ for all games $G \in \Gamma$.

Proof (cf. Peleg and Tijs, 1992). Proof is by induction to $\left|N^{G}\right|$, the number of players in $G \in \Gamma$. If $G$ is a 1-person game in $\Gamma$, the Nash equilibrium set consists of the points where the payoff function $U$ attains its maximum value (if anywhere). Then (UM) gives $\varphi(G) \subset \operatorname{NE}(G)$.

Suppose that the inclusion $\varphi(G) \subset \mathrm{NE}(G)$ was proved for games $G \in \Gamma$ with less than $n$ players. Let $G$ be a game in $\Gamma$ with $n$ players and $x \in \varphi(G)$. Then, by (CONS) for $\varphi, G^{S, x} \in \Gamma$ and $x_{S} \in \varphi\left(G^{S, x}\right)$ for all proper coalitions $S \subset N^{G}$. From the induction hypothesis we infer that $x_{S} \in \mathrm{NE}\left(G^{S, x}\right)$. By (COCONS) for NE (Peleg and Tijs, 1992), we find $x \in \mathrm{NE}(G)$.

Q.E.D.

The following proposition studies the conditions that (NEM), (UM), and (CONS) characterize the Nash equilibrium solution.

Proposition 3 (cf. Peleg et al., 1993). If $\varphi$ is a solution concept defined on a class $\Gamma$ and $\varphi$ has the properties (NEM), (UM) and (CONS), if for every game $G \in \Gamma$ and every Nash equilibrium $\hat{x} \in \mathrm{NE}(G)$ a game $H \in \Gamma$ can be constructed such that the following conditions are satisfied:

(i) $N^{H} \supset N^{G}$,

(ii) $y_{N^{G}}=\hat{x}$ for every $y \in \mathrm{NE}(H)$,

(iii) $H^{N^{G}, y}=G$ for every $y \in \mathrm{NE}(H)$,

then $\varphi=\mathrm{NE}$.

Proof. From Proposition 2 we know that $\varphi(G) \subset \mathrm{NE}(G)$ for all games $G \in \Gamma$. Suppose that $G \in \Gamma$ and $\hat{x} \in \operatorname{NE}(G)$. Take a game $H \in \Gamma$ satisfying the properties mentioned in the proposition. Take $y \in \varphi(H)(\varphi$ satisfies 
$(\mathrm{NEM}))$. Then $\hat{x}=y_{N^{G}} \in \varphi\left(H^{N^{G}, y}\right)$ by (CONS) and $H^{N^{G}, y}=G$. Therefore, $\hat{x} \in \varphi(G)$.

Q.E.D.

Proposition 3 can be extended to classes of games wherein the Nash equilibrium set is sometimes empty. Then there is no solution rule $\varphi$ satisfying (NEM), (UM), and (CONS) (cf. Proposition 2). But, if we replace (NEM) by restricted nonemptiness ( $\mathrm{r}-\mathrm{NEM}$ ) saying $\varphi(G) \neq \varnothing$ if $\mathrm{NE}(G) \neq$ $\varnothing$, we can prove the same result.

In the following section we prove that in the classes $\Gamma_{\mathrm{f}}$ and $\Gamma_{\mathrm{c}}$ the construction $(G, \hat{x}) \rightarrow H$ is possible.

\section{The Construction of $H$}

In this section we construct for each game $G \in \Gamma_{f}$ (of $\Gamma_{\mathrm{c}}$ ) and each Nash equilibrium $\hat{x} \in \operatorname{NE}(G)$ a game $H \in \Gamma_{\mathrm{f}}$ or $\Gamma_{\mathrm{c}}$ respectively, satisfying the conditions (i), (ii), and (iii) of Proposition 3.

(A) Let $G$ be the mixed extension of a finite game and let $\hat{x}$ be a Nash equilibrium of $G$. The game $H$ will have $3 n$ players where $n=\left|N^{G}\right|$. The player set $N^{H}$ consists of $\left\{i, i_{1}, i_{2} \mid i \in N^{G}\right\}$. The players $i, i_{1}$, and $i_{2}$ have the same strategy set $\Delta_{i}$ as player $i$ has in the game $G$.

The payoff functions of the players $i, i_{1}$, and $i_{2}$ are denoted by $U_{i}^{*}, V_{i}^{*}$, and $W_{i}^{*}$ and the strategies are called $x_{i}, y_{i}$, and $z_{i}$, respectively. The definition of the payoff functions is

$$
U_{i}^{*}(x, y, z):=U_{i}\left(x_{i} \mid x_{-i}+\hat{x}_{-i}-y_{-i}\right)-\left\langle x_{i}-\hat{x}_{i}, y_{i}-\hat{x}_{i}\right\rangle,
$$

where $x_{-i}+\hat{x}_{-i}-y_{-i}$ is a shorthand notation for $\left[\left(x_{j}+\hat{x}_{j}-y_{j}\right)_{j \neq i}\right]$ and $\langle.,$.$\rangle denotes the Euclidean inner product. Notice that x_{j}+\hat{x}_{j}-y_{j}$ is not necessarily an element of $\Delta_{j}$ but it is an element of the affine hull of $\Delta_{j}$. The multilinearity of $U_{i}$, the payoff functions in $G$, allows a unique multilinear extension of $U_{i}$ to affine hulls.

Furthermore, ${ }^{1}$

$$
V_{i}^{*}(x, y, z):=\left\langle y_{i}, x_{i}-z_{i}\right\rangle, \quad W_{i}^{*}(x, y, z):=\left\langle z_{i}, y_{i}-x_{i}\right\rangle .
$$

This finishes the definition of $H$. It is immediately clear that $H \in \Gamma_{\mathrm{f}}$.

\footnotetext{
${ }^{1}$ The referee pointed us to the following interpretation of the game $H$. Player $i_{1}$ obtains a positive payoff for as far as he matches the strategy of player $i$ and loses payoff for as far as he matches the strategy of player $i_{2}$. Player $i_{2}$ loses if he matches player $i$ and wins payoff if he matches the strategy of player $i_{1}$.
} 
We prove that all equilibria $(x, y, z)$ of $H$ satisfy $x=y=\hat{x}$. Then we find $(x, y, z)_{N^{G}}=x=\hat{x}$ and, if we substitute $y=\hat{x}, U_{i}^{*}(x, y, z)_{y=\hat{x}}=$ $U_{i}\left(x_{i} \mid x_{-i}\right)=U_{i}(x)$ for every player $i \in N^{G}$.

Suppose $(x, y, z)$ is a Nash equilibrium of $H$ and $x_{i} \neq y_{i}$ for some index $i \in N^{G}$.

If $z_{i j}>0$ (player $i_{2}$ puts positive weight on his $j$ th strategy), then $y_{i j}>$ $x_{i j}$. For, as $(x, y, z)$ is an equilibrium and $y_{i k}>x_{i k}$ for at least one pure strategy $k$, player $i_{2}$ uses only strategies $k$ with $y_{i k}-x_{i k}>0$. Then $y_{i j}>0$ and therefore $x_{i j} \geq z_{i j}$ by the same argument but now for player $i_{1}$. Then

$$
\sum_{j: z_{i j}>0} y_{i j}>\sum_{j: z_{i j}>0} x_{i j} \geq \sum_{j: z_{i j}>0} z_{i j}=1 .
$$

This is impossible: $x=y$.

Suppose that $x_{i}=y_{i} \neq \hat{x}_{i}$ for some $i \in N^{G}$. If player $i$ deviates from $x_{i}$ to $\hat{x}_{i}$, the value of his payoff goes from $U_{i}\left(x_{i} \mid \hat{x}_{-i}\right)-\left\|x_{i}-\hat{x}_{i}\right\|^{2}$ to $U_{i}(\hat{x})$. This is an improvement as $U_{i}(\hat{x}) \geq U_{i}\left(x_{i} \mid \hat{x}_{-i}\right)(\hat{x}$ is an equilibrium) and $\left\|x_{i}-\hat{x}_{i}\right\|^{2}>0$ if $x_{i} \neq \hat{x}_{i}$. Therefore, $x=y=\hat{x}$.

Q.E.D.

(B) Let $G$ be a strategic game with compact convex strategy spaces $\left\{A_{i}^{G}\right\}_{i \in N^{G}}$, subsets of a finite-dimensional vector space and payoff functions $\left\{U_{i}\right\}_{i \in N^{G}}$ satisfying:

(i) the functions $U_{i}: A^{G}=\prod_{i \in N^{G}} \mathrm{~A}_{i}^{G} \rightarrow R$ are continuous

(ii) the functions $U_{i}\left(\bullet \mid x_{-i}\right): x_{i} \in A_{i}^{G} \rightarrow U_{i}\left(x_{i} \mid x_{-i}\right)$ is concave for every complementary strategy profile $x_{-i} \in \Pi_{j \neq i} A_{j}^{G}$.

Let $\hat{x}$ be an equilibrium of $G$. To construct $H$ we take $N^{H}$ : = $\left\{i, i_{1} \mid i \in N^{G}\right\}$ and $A_{i}^{H}=A_{i_{1}}^{H}$ : $=A_{i}^{G}$. So, $H$ has $2 n$ players. Before we can introduce the payoff functions we need the following observations:

(1) The strategy space $A_{i}^{G}$ may be assumed to have the same dimension as the surrounding finite dimensional vector space $E_{i}$.

(2) If $x_{i}, \hat{x}_{i}$, and $y_{i}$ are elements of $A_{i}^{G}$, then $x_{i}+\hat{x}_{i}-y_{i}$ is perhaps not an element of $A_{i}^{G}$ but it is an element of $E_{i}$.

(3) There exists a continuous retraction of $E_{i}$ to $A_{i}^{G}$, i.e., there is a continuous map $\pi_{i}: E_{i} \rightarrow A_{i}^{G}$ with $\pi_{i}(x)=x$ for $x \in A_{i}^{G}$.

Let $(x, y, z)$ be a strategy profile in $H$. Using the shorthand notation $\pi_{-i}\left(x_{-i}+\hat{x}_{-i}+y_{-i}\right)$ for $\left(\pi_{j}\left(x_{j}+\hat{x}_{j}-y_{j}\right)_{j \neq i}\right)$, we define

$$
U_{i}^{*}(x, y,):=U_{i}\left(x_{i} \mid \pi_{-i}\left(x_{-i}+\hat{x}_{-i}-y_{-i}\right)\right)-\left\|x_{i}-\hat{x}_{i}\right\|\left\|y_{i}-\hat{x}_{i}\right\| .
$$

Note that $U_{i}^{*}$ is a continuous function and concave in $x_{i}$. For the other 
players $i_{1}$ the payoff functions are $V_{i}^{*}(x, y, z):=-\left\|y_{i}-x_{i}\right\|$. These functions are again continuous in $(x, y)$ and concave in $y_{i}$.

Suppose that $(x, y)$ is an equilibrium of $H$. Then $x=y$ (as follows from the maximality of the payoff $V_{i}^{*}$ ). If $x_{i} \neq \hat{x}_{i}$ for some $i \in N^{G}$, the deviation from $x_{i}$ to $\hat{x}_{i}$ gives player $i$ a change in payoff $U_{i}(\hat{x})-U_{i}\left(x_{i} \mid \hat{x}_{-i}\right)$ and $\left\|x_{i}-\hat{x}_{i}\right\|^{2}$. The first term is nonnegative, the second term is positive. So, if $x \neq \hat{x}$, we are not in an equilibrium. Therefore, the game $H$ has one equilibrium, namely $(x=\hat{x}, y=\hat{x}$ ). The properties (i), (ii), and (iii) of Proposition 3 can now be checked immediately.

Q.E.D.

If $\Gamma_{c}^{\prime}$ is the class of strategic games with compact convex strategy spaces and continuous payoff functions (so we delete the concavity condition), the same construction (B) can be used to prove

THEOREM 1.bis. If $\varphi$ is a solution rule defined on $\Gamma_{c}^{\prime}$ satisfying (r-NEM), (UM), and (CONS), then $\varphi=\mathrm{NE}$.

\section{ACKNOWLEDGMENTS}

The seminal idea of how to attack the problem discussed in this paper we owe to Bezalel Peleg. During the visit of one of the authors (JP) to the Hebrew University in Jerusalem he discussed the problem with Peleg and we tried to solve it for different classes of games (see the references Peleg et al., 1994, and Peleg and Sudhölter, 1994). We thank the Center of Rationality for their hospitality and Peleg for his stimulating cooperation.

\section{REFERENCES}

Aumann, R. J. (1959). "Acceptable Points in General Cooperative $n$-Person Games, in "Contributions to the Theory of Games IV" (A. W. Tucker and R. D. Luce, Eds.) pp. 287-324. Princeton, N. J.; Princeton Univ. Press.

Bernheim, B. D., Peleg, B., And Whinston, M. D. (1987). "Coalitional-Proof Nash Equilibria. I. Concepts," J. Econ. Theory 42, 1-12.

GlicksberG, I. L. (1952). "A Further Generalization of the Kakutani Fixed Point Theorem With Application to Nash Equilibrium Points," Proc. Amer. Math Soc. 3, 170-174.

Harsanyi, J. C. (1973a). "Games with Randomly Disturbed Payoffs: A New Rationale for Mixed Strategy Equilibrium Points, Int. J. Game Theory 2, 1-23.

Harsanyi, J. C. (1973b). Oddness of the number of equilibrium points: a new proof, Int. J. Game Theory 2, 235-250.

Harsanyi, J. C., And Selten, R. (1982). A General Theory of Equilibrium. Berlin: Springer-Verlag.

Kalai, E. And Samet, D. (1984) Persistent Equilibria. Int. J. of Game Theory 13, 129-144. Kohlberg, E., And Mertens, J.-F. (1986). “On the Strategic Stability of Equilibria,” Econometrica 54, 1003-1039. 
Myerson, R. B. (1978). "Refinements of the Nash Equilibrium Point Concept," Int. J. Game Theory 7, 73-80.

Nash, J. (1951). Non-cooperative games. Ann. of Math. 54, 286-295.

Peleg, B., And Tiss, S. H. (1992). "The Consistency Principle for Games in Strategic Form," Discussion Paper 23, Center for Rationality and Interactive Decision Theory. Jerulsalem: The Hebrew University.

Peleg, B., Potters, J. A. M., And Tiss, S. H. (1994). "Minimality of Consistent Solutions for Strategic Games, in Particular for Potential Games," Econ. Theory, forthcoming.

Peleg, B., And Sudholter, P. (1994). “An Axiomatization of Nash Equilibria in Economic Situation," discussion paper. University of Bielefeld.

Selten, R. (1975). "Reexamination of the Perfectness Concept for Equilibrium Points in Extensive Games, Int. J. Game Theory 4, 25-55.

Van Damme, E. E. C. (1987). Stability and Perfection of Nash Equilibria. Berlin: SpringerVerlag. 